\title{
The Split Feasibility Problems for Countable Families of Asymptotically Strict Pseudocontractions
}

\author{
Wei-Qi Deng \\ College of Statistics and Mathematics, Yunnan University of Finance and Economics, Kunming, Yunnan 650221, China \\ Correspondence should be addressed to Wei-Qi Deng; dwq1273@126.com
}

Received 15 May 2013; Accepted 22 August 2013

Academic Editor: Somyot Plubtieng

Copyright (C) 2013 Wei-Qi Deng. This is an open access article distributed under the Creative Commons Attribution License, which permits unrestricted use, distribution, and reproduction in any medium, provided the original work is properly cited.

An up-to-date algorithm for solving the split feasibility problem for countable families of asymptotically strict pseudocontractions is introduced in the framework of Hilbert spaces. Our results greatly improve and extend those of other authors whose related research studies are restricted to the situation of at most finitely many such mappings.

\section{Introduction}

The split feasibility problem (SFP) in finite dimensional spaces was first introduced by Censor and Elfving [1] for modeling inverse problems which arise from phase retrievals and in medical image reconstruction [2]. Recently, it has been found that the SFP can also be used in various disciplines such as image restoration, computer tomograph, and radiation therapy treatment planning [3-5].

The split feasibility problem in an infinite dimensional Hilbert space can be found in $[2,4,6-8]$.

Let $H_{1}$ and $H_{2}$ be two real Hilbert spaces with inner product $\langle\cdot,, \cdot\rangle$ and the corresponding norm $\|\cdot\|$. Let $C$ and $Q$ be nonempty closed convex subsets of $H_{1}$ and $H_{2}$, respectively. The purpose of this paper is to introduce and study the following multiple-set split feasibility problem for an infinite family of asymptotically strict pseudocontractions (MSSFP) in the framework of infinite-dimensional Hilbert spaces. Find $x^{*} \in C$ such that

$$
A x^{*} \in Q
$$

where $A: H_{1} \rightarrow H_{2}$ is a bounded linear operator.

In the sequel, we use $\Gamma$ to denote the set of solutions of the problem (MSSFP), that is,

$$
\Gamma=\{x \in C, A x \in Q\} .
$$

\section{Preliminaries}

We first recall some definitions, notations, and conclusions which will be needed in proving our main results.

Let $E$ be a Banach space. A mapping $T: E \rightarrow E$ is said to be demiclosed at origin, if for any sequence $\left\{x_{n}\right\} \subset E$ with $x_{n} \rightarrow x^{*}$ and $\left\|(I-T) x_{n}\right\| \rightarrow 0$, then $x^{*}=T x^{*}$, where $x_{n} \rightarrow$ $x^{*}$ denotes that $\left\{x_{n}\right\}$ converges weakly to $x^{*}$.

A Banach space $E$ is said to satisfy Opial's condition, if for any sequence $\left\{x_{n}\right\}$ in $E, x_{n} \rightarrow x^{*}$ implies that

$$
\begin{array}{r}
\liminf _{n \rightarrow \infty}\left\|x_{n}-x^{*}\right\|<\liminf _{n \rightarrow \infty}\left\|x_{n}-y\right\|, \quad \forall y \in E \\
\text { with } y \neq x^{*} .
\end{array}
$$

It is well known that every Hilbert space satisfies Opial's condition.

Definition 1. Let $H$ be a real Hilbert space, $T$ be a mapping from $H$ into itself and the fixed point set $F(T)$ of $T$ be nonempty.

(1) $T$ is called a $\left(\gamma,\left\{k_{n}\right\}\right)$-asymptotically strict pseudocontraction if there exists a constant $\gamma \in[0,1)$ and a sequence $\left\{k_{n}\right\} \subset[1, \infty)$ with $k_{n} \rightarrow 1$ such that

$$
\left\|T^{n} x-T^{n} y\right\|^{2} \leq k_{n}\|x-y\|^{2}+\gamma\left\|\left(I-T^{n}\right) x-\left(I-T^{n}\right) y\right\|^{2},
$$

$$
\forall x, y \in H \text {. }
$$


Especially, if $k_{n}=1$ for each $n \geq 1$ in (4) and there exists a $\gamma \in[0,1)$ such that

$$
\begin{array}{r}
\|T x-T y\|^{2} \leq\|x-y\|^{2}+\gamma\|(I-T) x-(I-T) y\|^{2}, \\
\forall x, y \in H,
\end{array}
$$

then $T: H \rightarrow H$ is called a $\gamma$-strict pseudocontraction.

(2) $T$ is said to be uniformly L-Lipschitzian if there exists a constant $L>0$ such that

$\left\|T^{n} x-T^{n} y\right\|^{2} \leq L\|x-y\|, \quad \forall x, y \in H, n \geq 1$.

(3) $T$ is said to be semicompact if for any bounded sequence $\left\{x_{n}\right\} \subset H$ with $\lim _{n \rightarrow \infty}\left\|x_{n}-T x_{n}\right\|=0$, there exists a subsequence $\left\{x_{n_{i}}\right\}$ of $\left\{x_{n}\right\}$ such that $\left\{x_{n_{i}}\right\}$ converges strongly to a point $x^{*} \in H$.

Remark 2. (1) If we put $\gamma=0$ in (4), then the mapping $T$ : $H \rightarrow H$ is asymptotically nonexpansive.

(2) If we put $\gamma=0$ in (5), then the mapping $T: H \rightarrow H$ is nonexpansive.

(3) Each $\left(\gamma,\left\{k_{n}\right\}\right)$-asymptotically strict pseudocontraction and each $\gamma$-strict pseudocontraction both are demiclosed at origin [9].

In 2011, Moudafi [10] proposed the following iterative algorithm for solving split common fixed problem of quasinonexpansive mappings: for arbitrarily chosen $x_{1} \in H_{1}$,

$$
\begin{gathered}
u_{n}=x_{n}+\gamma \beta A^{*}(T-I) A x_{n}, \\
x_{n+1}=\left(1-\alpha_{n}\right) u_{n}+\alpha_{n} U u_{n}, \quad n \in \mathbb{N},
\end{gathered}
$$

and proved that $\left\{x_{n}\right\}$ converges weakly to a split common fixed point $x^{*} \in \Gamma$, where $U: H_{1} \rightarrow H_{1}$ and $T: H_{2} \rightarrow H_{2}$ are two quasinonexpansive mappings, $A: H_{1} \rightarrow H_{2}$ is a bounded linear operator, and $A^{*}$ denotes the adjoint of $A$.

Motivated and inspired by the studies of Moudafi $[10,11]$ and Chang et al. [12], in this paper, we introduce an algorithm for solving the split feasibility problems for countable families of asymptotically strict pseudocontractions and prove some strong and weak convergence theorems for such mappings in Hilbert spaces. The results extend those of the authors [12] whose related research studies are restricted to the situation of at most finite families of such mappings.

By using the well-known inequality $\langle x, y\rangle=(1 / 2)\|x\|^{2}+$ $(1 / 2)\|y\|^{2}-(1 / 2)\|x-y\|^{2}$ in Hilbert spaces, we can easily show the following proposition, whose proof is omitted.

Proposition 3 (see [12]). Let $T: H \rightarrow H$ be $a\left(\gamma,\left\{k_{n}\right\}\right)$ asymptotically strict pseudocontraction. If $\Gamma \neq \emptyset$, then for each $p \in F(T)$ and $x \in H$, the following inequalities hold and they are equivalent:

$$
\begin{gathered}
\left\|T^{n} x-p\right\|^{2} \leq k_{n}\|x-p\|^{2}+\gamma\left\|x-T^{n} x\right\|^{2} \\
\left\langle x-T^{n} x, x-p\right\rangle \geq \frac{1-\gamma}{2}\left\|x-T^{n} x\right\|^{2}-\frac{k_{n}-1}{2}\|x-p\|^{2} ;
\end{gathered}
$$

$$
\left\langle x-T^{n} x, p-T^{n} x\right\rangle \leq \frac{1+\gamma}{2}\left\|x-T^{n} x\right\|^{2}+\frac{k_{n}-1}{2}\|x-p\|^{2} .
$$

Lemma 4 (see [13]). Let $\left\{a_{n}\right\},\left\{b_{n}\right\}$, and $\left\{\delta_{n}\right\}$ be the sequences of nonnegative real numbers satisfying

$$
a_{n+1} \leq\left(1+\delta_{n}\right) a_{n}+b_{n} .
$$

If $\sum_{n=1}^{\infty} \delta_{n}<\infty$ and $\sum_{n=1}^{\infty} b_{n}<\infty$, then the $\lim _{n \rightarrow \infty} a_{n}$ exists.

Lemma 5 (see [14]). Let $K$ be a nonempty closed convex subset of a real Hilbert space $H$ and $T$ a nonexpansive mapping from $K$ into itself. If $T$ has a fixed point, then $I-T$ is demiclosed at zero, where I is the identity mapping of $H$.

Lemma 6 (see [15]). The unique solutions to the positive integer equation

$$
n=i+\frac{(m-1) m}{2}, \quad m \geq i, n=1,2,3, \ldots
$$

are

$$
\begin{array}{r}
i=n-\frac{(m-1) m}{2}, \quad m=-\left[\frac{1}{2}-\sqrt{2 n+\frac{1}{4}}\right], \\
n=1,2,3, \ldots,
\end{array}
$$

where $[x]$ denotes the maximal integer that is not larger than $x$.

\section{Main Results}

In the sequel, we assume that the following conditions are satisfied:

(a) $H_{1}$ and $H_{2}$ are two real Hilbert spaces, $A: H_{1} \rightarrow$ $\mathrm{H}_{2}$ is a bounded linear operator, and $A^{*}$ denotes the adjoint of $A$;

(b) $\left\{S_{i}\right\}: H_{1} \rightarrow H_{1}$ is a sequence of uniformly $L_{1}$-Lipschitzian and $\left(\beta_{i},\left\{k_{1, n}^{(i)}\right\}\right)$-asymptotically strict pseudocontractions and $\left\{T_{i}\right\}: \mathrm{H}_{2} \rightarrow \mathrm{H}_{2}$ is a sequence of uniformly $L_{2}$-Lipschitzian and $\left(\mu_{i},\left\{k_{2, n}^{(i)}\right\}\right)$-asymptotically strict pseudocontractions satisfying the following conditions:

(1) $C:=\cap_{i=1}^{\infty} F\left(S_{i}\right) \neq \emptyset, Q:=\cap_{i=1}^{\infty} F\left(T_{i}\right) \neq \emptyset$;

(2) $\beta:=\sup _{i \geq 1}\left\{\beta_{i}\right\}<1$ and $\mu:=\sup _{i \geq 1}\left\{\mu_{i}\right\}<1$;

(3) for each $i \geq 1, k_{n}^{(i)}:=\max \left\{k_{1, n}^{(i)}, k_{2, n}^{(i)}\right\}$, and $\sum_{i=1}^{\infty} \sum_{n=1}^{\infty}\left(k_{n}^{(i)}-1\right)<\infty$. 
The multiple-set split feasibility problem for infinite families of nonlinear mappings $\left\{S_{i}\right\}$ and $\left\{T_{i}\right\}$ is to find a point

$$
q \in C \text { such that } A q \in Q
$$

whose set of solutions is denoted by $\Gamma$.

Lemma 7. Let $H_{1}, H_{2}, A,\left\{S_{i}\right\},\left\{T_{i}\right\}, C, Q, \beta, \mu, L_{1}, L_{2}$ and $\left\{k_{n}^{(i)}\right\}$ be the same as those mentioned above. Let $\left\{x_{n}\right\}$ be the following sequence generated by an arbitrarily chosen $x_{1} \in H_{1}$

$$
\begin{gathered}
u_{n}=x_{n}+\gamma A^{*}\left(\left(T_{n}^{*}\right)^{m_{n}}-I\right) A x_{n}, \\
x_{n+1}=\left(1-\alpha_{n}\right) u_{n}+\alpha_{n}\left(S_{n}^{*}\right)^{m_{n}} u_{n}, \quad n \in \mathbb{N},
\end{gathered}
$$

where $T_{n}^{*}=T_{i_{n}}, S_{n}^{*}=S_{i_{n}}$ with $i_{n}$ and $m_{n}$ being the solutions to the positive integer equation: $n=i+(m-1) m / 2(m \geq i, n=$ $1,2, \ldots)$; that is, for each $n \geq 1$, there exist unique $i_{n}$ and $m_{n}$ such that

$$
\begin{gathered}
i_{1}=1, i_{2}=1, i_{3}=2, i_{4}=1, \\
i_{5}=2, i_{6}=3, i_{7}=1, i_{8}=2, \ldots ; \\
m_{1}=1, m_{2}=2, m_{3}=2, m_{4}=3, m_{5}=3, \\
m_{6}=3, m_{7}=4, m_{8}=4, \ldots ;
\end{gathered}
$$

$\left\{\alpha_{n}\right\}$ is a sequence in $[0,1]$, and $\gamma$ is a constant satisfying the following condition:

(4): $\alpha_{n} \in(\delta, 1-\beta)$ for all $n \geq 1$ and $\gamma \in\left(0,(1-\mu) /\|A\|^{2}\right)$, where $\delta \in(0,1-\beta)$ is a positive constant. If $\Gamma \neq \emptyset$, for any $q \in \Gamma$, then

(I) $\lim _{n \rightarrow \infty}\left\|x_{n}-q\right\|$ and $\lim _{n \rightarrow \infty}\left\|u_{n}-q\right\|$ exist and have the same values;

(II) for each $i \geq 1$, there exists a corresponding subsequence $\left\{x_{n}\right\}_{n \in \mathbb{N}_{i}}$ of $\left\{x_{n}\right\}$ such that

$$
\lim _{\mathbb{N}_{i} \ni n \rightarrow \infty}\left\|u_{n}-S_{i} u_{n}\right\|=\left\|A x_{n}-T_{i} A x_{n}\right\|=0,
$$

where $\mathbb{N}_{i}:=\{n \in \mathbb{N}: n=i+(m-1) m / 2, m \geq i, m \in \mathbb{N}\}$.

Proof. (I) Taking $q \in \Gamma$, that is, $q \in C$ and $A q \in Q$, and using (15) and (9), we have

$$
\begin{aligned}
\left\|x_{n+1}-q\right\|^{2} \leq & \left\|u_{n}-q-\alpha_{n}\left(u_{n}-\left(S_{n}^{*}\right)^{m_{n}} u_{n}\right)\right\|^{2} \\
= & \left\|u_{n}-q\right\|^{2}-2 \alpha_{n}\left\langle u_{n}-q, u_{n}-\left(S_{n}^{*}\right)^{m_{n}} u_{n}\right\rangle \\
& +\alpha_{n}^{2}\left\|u_{n}-\left(S_{n}^{*}\right)^{m_{n}} u_{n}\right\|^{2} \leq\left\|u_{n}-q\right\|^{2} \\
& -\alpha_{n}\left[(1-\beta)\left\|u_{n}-\left(S_{n}^{*}\right)^{m_{n}} u_{n}\right\|^{2}\right. \\
& \left.-\left(k_{m_{n}}^{\left(i_{n}\right)}-1\right)\left\|u_{n}-q\right\|^{2}\right] \\
& +\alpha_{n}^{2}\left\|u_{n}-\left(S_{n}^{*}\right)^{m_{n}} u_{n}\right\|^{2}
\end{aligned}
$$

$$
\begin{aligned}
= & \left(1+\alpha_{n}\left(k_{m_{n}}^{\left(i_{n}\right)}-1\right)\right)\left\|u_{n}-q\right\|^{2} \\
& -\alpha_{n}\left(1-\beta-\alpha_{n}\right)\left\|u_{n}-\left(S_{n}^{*}\right)^{m_{n}} u_{n}\right\|^{2},
\end{aligned}
$$

$$
\begin{aligned}
\left\|u_{n}-q\right\|^{2}= & \left\|x_{n}+\gamma A^{*}\left(\left(T_{n}^{*}\right)^{m_{n}}-I\right) A x_{n}-q\right\|^{2} \\
= & \left\|x_{n}-q\right\|^{2}+\gamma^{2}\left\|A^{*}\left(\left(T_{n}^{*}\right)^{m_{n}}-I\right) A x_{n}\right\|^{2} \\
& +2 \gamma\left\langle x_{n}-q, A^{*}\left(\left(T_{n}^{*}\right)^{m_{n}}-I\right) A x_{n}\right\rangle \\
= & \left\|x_{n}-q\right\|^{2} \\
& +\gamma^{2}\left\langle\left(\left(T_{n}^{*}\right)^{m_{n}}-I\right) A x_{n}, A A^{*}\left(\left(T_{n}^{*}\right)^{m_{n}}-I\right) A x_{n}\right\rangle \\
& +2 \gamma\left\langle x_{n}-q, A^{*}\left(\left(T_{n}^{*}\right)^{m_{n}}-I\right) A x_{n}\right\rangle,
\end{aligned}
$$

where

$$
\begin{aligned}
& \gamma^{2}\left\langle\left(\left(T_{n}^{*}\right)^{m_{n}}-I\right) A x_{n}, A A^{*}\left(\left(T_{n}^{*}\right)^{m_{n}}-I\right) A x_{n}\right\rangle \\
& \leq\|A\|^{2} \gamma^{2}\left\langle\left(\left(T_{n}^{*}\right)^{m_{n}}-I\right) A x_{n},\left(\left(T_{n}^{*}\right)^{m_{n}}-I\right) A x_{n}\right\rangle \\
& \leq\|A\|^{2} \gamma^{2}\left\|\left(\left(T_{n}^{*}\right)^{m_{n}}-I\right) A x_{n}\right\|^{2}, \\
& 2 \gamma\left\langle x_{n}-q, A^{*}\left(\left(T_{n}^{*}\right)^{m_{n}}-I\right) A x_{n}\right\rangle \\
& =2 \gamma\left\langle A\left(x_{n}-q\right),\left(\left(T_{n}^{*}\right)^{m_{n}}-I\right) A x_{n}\right\rangle \\
& =2 \gamma\left\langle A\left(x_{n}-q\right)+\left(\left(T_{n}^{*}\right)^{m_{n}}-I\right) A x_{n}\right. \\
& \left.\quad-\left(\left(T_{n}^{*}\right)^{m_{n}}-I\right) A x_{n},\left(\left(T_{n}^{*}\right)^{m_{n}}-I\right) A x_{n}\right\rangle \\
& =2 \gamma\left(\left\langle\left(T_{n}^{*}\right)^{m_{n}} A x_{n}-A q,\left(\left(T_{n}^{*}\right)^{m_{n}}-I\right) A x_{n}\right\rangle\right. \\
& \left.\quad-\left\|\left(\left(T_{n}^{*}\right)^{m_{n}}-I\right) A x_{n}\right\|^{2}\right) .
\end{aligned}
$$

Further, letting $x=A x_{n}, T^{n}=\left(T_{n}^{*}\right)^{m_{n}}, p=A q, \gamma=\mu$ in (10) and noting $A q \in F\left(T_{n}^{*}\right)$, we have

$$
\begin{aligned}
& \left\langle\left(T_{n}^{*}\right)^{m_{n}} A x_{n}-A q,\left(\left(T_{n}^{*}\right)^{m_{n}}-I\right) A x_{n}\right\rangle \\
& \leq \frac{1+\mu}{2}\left\|\left(\left(T_{n}^{*}\right)^{m_{n}}-I\right) A x_{n}\right\|^{2}+\frac{k_{m_{n}}^{\left(i_{n}\right)}-1}{2}\left\|A x_{n}-A q\right\|^{2} \\
& \leq \frac{1+\mu}{2}\left\|\left(\left(T_{n}^{*}\right)^{m_{n}}-I\right) A x_{n}\right\|^{2}+\frac{\left(k_{m_{n}}^{\left(i_{n}\right)}-1\right)\|A\|^{2}}{2}\left\|x_{n}-q\right\|^{2} .
\end{aligned}
$$

Substituting (22) into (21) and simplifying it, we have

$$
\begin{gathered}
2 \gamma\left\langle x_{n}-q, A^{*}\left(\left(T_{n}^{*}\right)^{m_{n}}-I\right) A x_{n}\right\rangle \\
\leq \gamma(\mu-1)\left\|\left(\left(T_{n}^{*}\right)^{m_{n}}-I\right) A x_{n}\right\|^{2} \\
+\left(k_{m_{n}}^{\left(i_{n}\right)}-1\right) \gamma\|A\|^{2}\left\|x_{n}-q\right\|^{2} .
\end{gathered}
$$


Substituting (20) and (23) into (19) and simplifying it, we have

$$
\begin{aligned}
\left\|u_{n}-q\right\|^{2} \leq & \left\|x_{n}-q\right\|^{2}+\gamma^{2}\|A\|^{2}\left\|\left(\left(T_{n}^{*}\right)^{m_{n}}-I\right) A x_{n}\right\|^{2} \\
& +\gamma(\mu-1)\left\|\left(\left(T_{n}^{*}\right)^{m_{n}}-I\right) A x_{n}\right\|^{2} \\
& +\left(k_{m_{n}}^{\left(i_{n}\right)}-1\right) \gamma\|A\|^{2}\left\|x_{n}-q\right\|^{2} \\
= & \left\|x_{n}-q\right\|^{2}-\gamma\left(1-\mu-\gamma\|A\|^{2}\right) \\
& \times\left\|\left(\left(T_{n}^{*}\right)^{m_{n}}-I\right) A x_{n}\right\|^{2} \\
& +\left(k_{m_{n}}^{\left(i_{n}\right)}-1\right) \gamma\|A\|^{2}\left\|x_{n}-q\right\|^{2} .
\end{aligned}
$$

Again, substituting (24) into (18) and simplifying it, we have

$$
\begin{aligned}
\left\|x_{n+1}-q\right\|^{2} \leq & \left(1+\alpha_{n}\left(k_{m_{n}}^{\left(i_{n}\right)}-1\right)\right) \\
& \times\left\{\left\|x_{n}-q\right\|^{2}\right. \\
& -\gamma\left(1-\mu-\gamma\|A\|^{2}\right) \\
& \times\left\|\left(\left(T_{n}^{*}\right)^{m_{n}}-I\right) A x_{n}\right\|^{2} \\
& \left.+\left(k_{m_{n}}^{\left(i_{n}\right)}-1\right) \gamma\|A\|^{2}\left\|x_{n}-q\right\|^{2}\right\} \\
& -\alpha_{n}\left(1-\beta-\alpha_{n}\right)\left\|u_{n}-\left(S_{n}^{*}\right)^{m_{n}} u_{n}\right\|^{2} \\
\leq & \left(1+\alpha_{n}\left(k_{m_{n}}^{\left(i_{n}\right)}-1\right)\right)\left\|x_{n}-q\right\|^{2} \\
& -\gamma\left(1-\mu-\gamma\|A\|^{2}\right)\left\|\left(\left(T_{n}^{*}\right)^{m_{n}}-I\right) A x_{n}\right\|^{2} \\
+ & \left(1+\alpha_{n}\left(k_{m_{n}}^{\left(i_{n}\right)}-1\right)\right)\left(k_{m_{n}}^{\left(i_{n}\right)}-1\right) \gamma\|A\|^{2}\left\|x_{n}-q\right\|^{2} \\
& -\alpha_{n}\left(1-\beta-\alpha_{n}\right)\left\|u_{n}-\left(S_{n}^{*}\right)^{m_{n}} u_{n}\right\|^{2} .
\end{aligned}
$$

By condition (4), we have

$$
\begin{aligned}
\left\|x_{n+1}-q\right\|^{2} \leq & \left(1+\alpha_{n}\left(k_{m_{n}}^{\left(i_{n}\right)}-1\right)\right)\left\|x_{n}-q\right\|^{2} \\
& +\left(1+\alpha_{n}\left(k_{m_{n}}^{\left(i_{n}\right)}-1\right)\right)\left(k_{m_{n}}^{\left(i_{n}\right)}-1\right) \\
& \times \gamma\|A\|^{2}\left\|x_{n}-q\right\|^{2} \\
\leq & \left(1+K\left(k_{m_{n}}^{\left(i_{n}\right)}-1\right)\right)\left\|x_{n}-q\right\|^{2},
\end{aligned}
$$

where

$$
K=\sup _{n \geq 1}\left\{\alpha_{n}+\left(1+\alpha_{n}\left(k_{m_{n}}^{\left(i_{n}\right)}-1\right)\right) \gamma\|A\|^{2}\right\}<\infty .
$$

Note that $\sum_{n=1}^{\infty}\left(k_{m_{n}}^{\left(i_{n}\right)}-1\right)=\sum_{i=1}^{\infty} \sum_{n=i}^{\infty}\left(k_{n}^{(i)}-1\right) \leq$ $\sum_{i=1}^{\infty} \sum_{n=1}^{\infty}\left(k_{n}^{(i)}-1\right)<\infty$. Hence, from Lemma 4 , we know that the following limit exists:

$$
\lim _{n \rightarrow \infty}\left\|x_{n}-q\right\|
$$

We now prove that for each $q \in \Gamma$, the limit

$$
\lim _{n \rightarrow \infty}\left\|u_{n}-q\right\|
$$

exists. In fact, from (25) and (28), it follows that

$$
\begin{aligned}
\gamma(1- & \left.\mu-\gamma\|A\|^{2}\right)\left\|\left(\left(T_{n}^{*}\right)^{m_{n}}-I\right) A x_{n}\right\|^{2} \\
& +\alpha_{n}\left(1-\beta-\alpha_{n}\right)\left\|u_{n}-\left(S_{n}^{*}\right)^{m_{n}} u_{n}\right\|^{2} \\
\leq & \left\|x_{n}-q\right\|^{2}-\left\|x_{n+1}-q\right\|^{2} \\
& +K\left(k_{m_{n}}^{\left(i_{n}\right)}-1\right)\left\|x_{n}-q\right\|^{2} \longrightarrow 0 \quad(n \longrightarrow \infty) .
\end{aligned}
$$

This, combined with condition (4), implies that

$$
\begin{gathered}
\lim _{n \rightarrow \infty}\left\|u_{n}-\left(S_{n}^{*}\right)^{m_{n}} u_{n}\right\|=0, \\
\lim _{n \rightarrow \infty}\left\|\left(\left(T_{n}^{*}\right)^{m_{n}}-I\right) A x_{n}\right\|=0 .
\end{gathered}
$$

Therefore, it follows from (19), (28), and (32) that $\lim _{n \rightarrow \infty}\left\|u_{n}-q\right\|$ exists.

(II) We firstly prove that $\lim _{n \rightarrow \infty}\left\|x_{n+1}-x_{n}\right\|=0$ and $\lim _{n \rightarrow \infty}\left\|u_{n+1}-u_{n}\right\|=0$. As a matter of fact, it follows from (15) that

$$
\begin{aligned}
\left\|x_{n+1}-x_{n}\right\|= & \left\|\left(1-\alpha_{n}\right) u_{n}+\alpha_{n}\left(S_{n}^{*}\right)^{m_{n}} u_{n}-x_{n}\right\| \\
= & \|\left(1-\alpha_{n}\right)\left(x_{n}+\gamma A^{*}\left(\left(T_{n}^{*}\right)^{m_{n}}-I\right) A x_{n}\right) \\
& +\alpha_{n}\left(\left(S_{n}^{*}\right)^{m_{n}} u_{n}-x_{n}\right) \| \\
= & \|\left(1-\alpha_{n}\right) \gamma A^{*}\left(\left(T_{n}^{*}\right)^{m_{n}}-I\right) A x_{n} \\
& +\alpha_{n}\left(\left(S_{n}^{*}\right)^{m_{n}} u_{n}-x_{n}\right) \| \\
= & \|\left(1-\alpha_{n}\right) \gamma A^{*}\left(\left(T_{n}^{*}\right)^{m_{n}}-I\right) A x_{n} \\
& +\alpha_{n}\left(\left(S_{n}^{*}\right)^{m_{n}} u_{n}-u_{n}\right) \\
& +\alpha_{n}\left(u_{n}-x_{n}\right) \| \\
= & \|\left(1-\alpha_{n}\right) \gamma A^{*}\left(\left(T_{n}^{*}\right)^{m_{n}}-I\right) A x_{n} \\
& +\alpha_{n}\left(\left(S_{n}^{*}\right)^{m_{n}} u_{n}-u_{n}\right) \\
& +\alpha_{n} \gamma A^{*}\left(\left(T_{n}^{*}\right)^{m_{n}}-I\right) A x_{n} \| \\
= & \| A^{*}\left(\left(T_{n}^{*}\right)^{m_{n}}-I\right) A x_{n} \\
& +\alpha_{n}\left(\left(S_{n}^{*}\right)^{m_{n}} u_{n}-u_{n}\right) \| .
\end{aligned}
$$

In view of (31) and (32), we have that

$$
\lim _{n \rightarrow \infty}\left\|x_{n+1}-x_{n}\right\|=0
$$


Similarly, it follows from (15), (32), and (34) that

$$
\begin{aligned}
\left\|u_{n+1}-u_{n}\right\|= & \| x_{n+1}+\gamma A^{*}\left(\left(T_{n+1}^{*}\right)^{m_{n+1}}-I\right) A x_{n+1} \\
& -\left(x_{n}+\gamma A^{*}\left(\left(T_{n}^{*}\right)^{m_{n}}-I\right) A x_{n}\right) \| \\
= & \left\|x_{n+1}-x_{n}\right\|+\gamma\left\|A^{*}\left(\left(T_{n+1}^{*}\right)^{m_{n+1}}-I\right) A x_{n+1}\right\| \\
& +\gamma\left\|A^{*}\left(\left(T_{n}^{*}\right)^{m_{n}}-I\right) A x_{n}\right\| \longrightarrow 0 \quad(n \longrightarrow \infty) .
\end{aligned}
$$

Next, for each $i \in \mathbb{N}$, we consider the corresponding subsequence $\left\{u_{n}\right\}_{n \in \mathbb{N}_{i}}$ of $\left\{u_{n}\right\}$. For example, by Lemma 6 and the definition of $\mathbb{N}_{1}$, we have $\mathbb{N}_{1}=\{1,2,4,7,11,16, \ldots\}$ and $i_{1}=i_{2}=i_{4}=i_{7}=i_{11}=i_{16}=\cdots=1$. Note that $\left\{m_{n}\right\}_{n \in \mathbb{N}_{i}}=\{i, i+1, i+2, \ldots\}$, that is, $m_{n}-1=m_{n-1}$, and $S_{n}^{*}=S_{i_{n}}=S_{i}$ whenever $n \in \mathbb{N}_{i}$. Set $\eta_{n}:=\left\|u_{n}-S_{i}^{m_{n}} u_{n}\right\|$. Since $\left\{S_{i}\right\}$ are uniformly $L_{1}$-Lipschitzian and $m_{n} \geq 1$ for $n \geq 1$, we have, for each $n \in \mathbb{N}_{i}$ and $n \geq 2$,

$$
\begin{aligned}
&\left\|u_{n}-S_{i} u_{n}\right\| \leq\left\|u_{n}-S_{i}^{m_{n}} u_{n}\right\|+\left\|S_{i}^{m_{n}} u_{n}-S_{i} u_{n}\right\| \\
& \leq \eta_{n}+L_{1}\left\|S_{i}^{m_{n}-1} u_{n}-u_{n}\right\| \\
& \leq \eta_{n}+L_{1}\left(\left\|S_{i}^{m_{n}-1} u_{n}-S_{i}^{m_{n}-1} u_{n-1}\right\|\right. \\
&\left.\quad+\left\|S_{i}^{m_{n}-1} u_{n-1}-u_{n}\right\|\right) \\
& \leq \eta_{n}+L_{1}\left\|S_{i}^{m_{n}-1} u_{n}-S_{i}^{m_{n}-1} u_{n-1}\right\| \\
& \quad+L_{1}\left(\left\|S_{i}^{m_{n}-1} u_{n-1}-u_{n-1}\right\|\right. \\
&\left.\quad+\left\|u_{n}-u_{n-1}\right\|\right) \\
& \leq \eta_{n}+L_{1}^{2}\left\|u_{n}-u_{n-1}\right\| \\
& \quad+L_{1}\left(\left\|S_{i}^{m_{n}-1} u_{n-1}-u_{n-1}\right\|+\left\|u_{n-1}-u_{n}\right\|\right) \\
& \leq \eta_{n}+L_{1}\left(1+L_{1}\right)\left\|u_{n}-u_{n-1}\right\|+L_{1} \eta_{n-1} .
\end{aligned}
$$

Thus, it follows from (31) and (35) that, for each $i \geq 1$,

$$
\lim _{\mathbb{N}_{i} \ni n \rightarrow \infty}\left\|u_{n}-S_{i} u_{n}\right\|=0 .
$$

Similarly, we have, for each $i \geq 1$,

$$
\lim _{\mathbb{N}_{i} \ni n \rightarrow \infty}\left\|A x_{n}-T_{i} A x_{n}\right\|=0 .
$$

This completes the proof.

Theorem 8. Let $H_{1}, H_{2}, A,\left\{S_{i}\right\},\left\{T_{i}\right\}, C, Q, \beta, \mu, L_{1}, L_{2}$ and $\left\{k_{n}^{(i)}\right\}$ be the same as those in Lemma 7. Suppose that $\left\{x_{n}\right\}$ is a sequence defined by (15). If $\Gamma \neq \emptyset$ and there exist mappings $S_{i_{0}} \in\left\{S_{i}\right\}_{i=1}^{\infty}$ and $T_{i_{0}} \in\left\{T_{i}\right\}_{i=1}^{\infty}$ and nondecreasing functions $f, h:[0, \infty) \rightarrow[0, \infty)$ with $f(0)=h(0)=0$ and $f(r), h(r)>$ 0 for all $r \in(0, \infty)$ such that $f\left(d\left(x_{n}, \Gamma\right)\right) \leq\left\|u_{n}-S_{i_{0}} u_{n}\right\|$ and $h\left(d\left(A x_{n}, Q\right)\right) \leq\left\|A x_{n}-T_{i_{0}} A x_{n}\right\|$ for all $n \geq 1$, then $\left\{x_{n}\right\}$ converges strongly to some member of $\Gamma$.
Proof. By Lemma 7, there exists a subsequence $\left\{u_{n}\right\}_{n \in \mathbb{N}_{i_{0}}}$ of $\left\{u_{n}\right\}$ such that

$$
\lim _{\mathbb{N}_{i_{0}} \ni n \rightarrow \infty}\left\|u_{n}-S_{i_{0}} u_{n}\right\|=0 .
$$

Since for all $n \in \mathbb{N}_{i_{0}}$,

$$
f\left(d\left(x_{n}, \Gamma\right)\right) \leq\left\|u_{n}-S_{i_{0}} u_{n}\right\|
$$

by taking limsup as $\mathbb{N}_{i_{0}} \ni n \rightarrow \infty$ on both sides in the inequality above, we have

$$
\lim _{\mathbb{N}_{i_{0}} \ni n \rightarrow \infty} f\left(d\left(x_{n}, \Gamma\right)\right)=0,
$$

which implies $\lim _{\mathbb{N}_{i_{0}} \ni n \rightarrow \infty} d\left(x_{n}, \Gamma\right)=0$ by the definition of the function $f$.

Now we will show that $\left\{x_{n}\right\}_{n \in \mathbb{N}_{i}}$ is a Cauchy sequence. By Lemma 7, there exists a constant $M>0$ such that $\left\|x_{n}-q\right\|^{2} \leq$ $M\left\|x_{m}-q\right\|^{2}$ for any $q \in \Gamma$ and all $n>m$. And for any $\epsilon>0$, there exists a positive integer $N$ such that $d^{2}\left(x_{n}, \Gamma\right)<\epsilon / 4 M$ for all $n \geq N$ and $n \in \mathbb{N}_{i_{0}}$. Then, for any $q \in \Gamma$ and $n, m \geq N$ and $n, m \in \mathbb{N}_{i_{0}}$, we have

$$
\begin{aligned}
\left\|x_{n}-x_{m}\right\|^{2} & \leq 2\left(\left\|x_{n}-q\right\|^{2}+\left\|x_{m}-q\right\|^{2}\right) \\
& \leq 4 M\left\|x_{N}-q\right\|^{2} .
\end{aligned}
$$

Taking the infimum in the above inequalities for all $q \in \Gamma$ yields that

$$
\left\|x_{n}-x_{m}\right\|^{2} \leq 4 M d^{2}\left(x_{N}, \Gamma\right)<\epsilon,
$$

which implies that $\left\{x_{n}\right\}_{n \in \mathbb{N}_{i}}$ is a Cauchy sequence. Therefore, there exists a $p \in H_{1}$ such that $x_{n} \rightarrow p$ as $\mathbb{N}_{i_{0}} \ni n \rightarrow$ $\infty$ since $H_{1}$ is complete. Firstly, we show that $p \in C$. $\lim _{\mathbb{N}_{i_{0}} \ni n \rightarrow \infty} d\left(x_{n}, \Gamma\right)=0$ shows that $d(p, \Gamma)=0$, which implies that $p \in C$ since $\Gamma \subset C$. Secondly, we show that $A p \in Q$. Since $\left\{x_{n}\right\}_{n \in \mathbb{N}_{i_{0}}}$ converges to $p$ and $h\left(d\left(A x_{n}, Q\right)\right) \leq$ $\left\|A x_{n}-T_{i_{0}} A x_{n}\right\|$ for all $n \in \mathbb{N}_{i_{0}}$, then $d(A p, Q)=0$. This implies that $A p \in Q$ because of the closedness of $Q$, and so $p \in \Gamma$. It finally follows from the existence of $\lim _{n \rightarrow \infty}\left\|x_{n}-p\right\|$ that $x_{n} \rightarrow p$ as $n \rightarrow \infty$. This completes the proof.

Example 9. Let $H_{1}=H_{2}=\mathbb{R}^{1}$ with the standard norm $\|\cdot\|=$ $|\cdot|$ and $C=[-1,1]$. Let $\left\{S_{i}\right\}=\left\{T_{i}\right\}: C \rightarrow C$ be two sequences of mappings defined by

$$
S_{i} x= \begin{cases}\frac{x}{i+1}, & x \in(0,1], \\ x, & x \in[-1,0]\end{cases}
$$

It is easily shown that $\left\{S_{i}\right\}$ is uniformly $L$-Lipschitzian and a sequence of $\left(0,\left\{k_{n}=1\right\}\right)$-asymptotically strict pseudocontractions. We now prove that the sequence $\left\{x_{n}\right\}$ defined by (15) converges strongly to some member of $\Gamma$. Let 
$A x=A^{*} x=x / 2$ for all $x \in C$ with $\|A\|=1 / 2$ and $\gamma=3 \in\left(0,1 /\|A\|^{2}\right)$. If $\left\{x_{n}\right\} \subset(0,1]$, we then have

$$
\begin{aligned}
\left|u_{n}-S_{1} u_{n}\right| & =\frac{1}{2}\left|u_{n}\right| \\
& =\frac{1}{2}\left|x_{n}+\frac{3}{2}\left[\left(\frac{1}{i_{n}+1}\right)^{m_{n}} \frac{x_{n}}{2}-\frac{x_{n}}{2}\right]\right| \\
& =\kappa_{n}\left|x_{n}\right|,
\end{aligned}
$$

where $\kappa_{n}:=(1 / 2)\left|1+(3 / 4)\left[\left(1 /\left(i_{n}+1\right)\right)^{m_{n}}-1\right]\right|$ with $\kappa:=$ $\inf _{n \geq 1} \kappa_{n}>0$. Define a nondecreasing function $f:[0, \infty) \rightarrow$ $[0, \infty)$ by $f(x)=\kappa x$. Since $\Gamma=[-1,0]$, we then have

$$
f\left(d\left(x_{n}, \Gamma\right)\right)=f\left(\left|x_{n}\right|\right)=\kappa\left|x_{n}\right| \leq\left|u_{n}-S_{1} u_{n}\right| .
$$

Similarly, we also can define a nondecreasing function $h$ : $[0, \infty) \rightarrow[0, \infty)$ with $h(0)=0$ such that

$$
h\left(d\left(A x_{n}, Q\right)\right) \leq\left|A x_{n}-T_{i_{0}} A x_{n}\right|
$$

for some $i_{0} \geq 1$, which implies that, by Lemma 7 and Theorem $8, x_{n} \rightarrow x^{*} \in \Gamma$ as $n \rightarrow \infty$.

Theorem 10. Let $H_{1}, H_{2}, A, C$ and $Q$ be the same as those in Lemma 7. Let $\left\{S_{i}\right\}: H_{1} \rightarrow H_{1}$ and $\left\{T_{i}\right\}: H_{2} \rightarrow H_{2}$ be two sequences of nonexpansive mappings. Let $\left\{x_{n}\right\}$ be the following sequence generated by an arbitrarily chosen $x_{1} \in H_{1}$

$$
\begin{gathered}
u_{n}=x_{n}+\gamma A^{*}\left(T_{n}^{*}-I\right) A x_{n}, \\
x_{n+1}=\left(1-\alpha_{n}\right) u_{n}+\alpha_{n} S_{n}^{*} u_{n}, \quad n \geq 1,
\end{gathered}
$$

where $\left\{\alpha_{n}\right\}$ is a sequence in $[\alpha, 1-\alpha]$ for some $\alpha \in(0,1) ; \gamma \in$ $\left(0,1 /\|A\|^{2}\right) ; T_{n}^{*}=T_{i_{n}}, S_{n}^{*}=S_{i_{n}}$ with $i_{n}$ satisfying the positive integer equation: $n=i+(m-1) m / 2(m \geq i, n=1,2, \ldots)$. Then $\left\{x_{n}\right\}$ converges weakly to an $x^{*} \in \Gamma$.

Proof. It is clear that both $\left\{S_{i}\right\}$ and $\left\{T_{i}\right\}$ are asymptotically strict pseudocontractions. Then, by the proof of Lemma 7, we have

$$
\begin{gathered}
\lim _{n \rightarrow \infty}\left\|u_{n}-S_{n}^{*} u_{n}\right\|=0, \\
\lim _{n \rightarrow \infty}\left\|\left(T_{n}^{*}-I\right) A x_{n}\right\|=0 .
\end{gathered}
$$

In addition, we also have

$$
\lim _{n \rightarrow \infty}\left\|u_{n+1}-u_{n}\right\|=\left\|x_{n+1}-x_{n}\right\|=0,
$$

which implies that, by induction, for any nonnegative integer $k$,

$$
\lim _{n \rightarrow \infty}\left\|u_{n+k}-u_{n}\right\|=\lim _{n \rightarrow \infty}\left\|x_{n+k}-x_{n}\right\|=0 .
$$

For each $k \geq 1$, since

$$
\begin{aligned}
\left\|u_{n}-S_{n+k}^{*} u_{n}\right\| \leq & \left\|u_{n}-u_{n+k}\right\|+\left\|u_{n+k}-S_{n+k}^{*} u_{n}\right\| \\
\leq & \left\|u_{n}-u_{n+k}\right\|+\left\|u_{n+k}-S_{n+k}^{*} u_{n+k}\right\| \\
& \quad+\left\|S_{n+k}^{*} u_{n+k}-S_{n+k}^{*} u_{n}\right\| \\
\leq & 2\left\|u_{n}-u_{n+k}\right\|+\left\|u_{n+k}-S_{n+k}^{*} u_{n+k}\right\|,
\end{aligned}
$$

it follows from (49) and (52) that

$$
\lim _{n \rightarrow \infty}\left\|u_{n}-S_{n+k}^{*} u_{n}\right\|=0 \text {. }
$$

Now, for each $i \geq 1$, we claim that

$$
\lim _{n \rightarrow \infty}\left\|u_{n}-S_{i} u_{n}\right\|=0
$$

As a matter of fact, setting

$$
n=N_{m}+i,
$$

where $N_{m}=(m-1) m / 2, m \geq i$, we obtain that

$$
\begin{aligned}
\left\|u_{n}-S_{i} u_{n}\right\| \leq & \left\|u_{n}-u_{N_{m}}\right\|+\left\|u_{N_{m}}-S_{i} u_{n}\right\| \\
\leq & \left\|u_{n}-u_{N_{m}}\right\|+\left\|u_{N_{m}}-S_{N_{m}+i}^{*} u_{N_{m}}\right\| \\
& +\left\|S_{N_{m}+i}^{*} u_{N_{m}}-S_{i} u_{n}\right\| \\
= & \left\|u_{n}-u_{N_{m}}\right\|+\left\|u_{N_{m}}-S_{N_{m}+i}^{*} u_{N_{m}}\right\| \\
& +\left\|S_{i} u_{N_{m}}-S_{i} u_{n}\right\| \\
\leq & 2\left\|u_{n}-u_{N_{m}}\right\|+\left\|u_{N_{m}}-S_{N_{m}+i}^{*} u_{N_{m}}\right\| \\
= & 2\left\|u_{n}-u_{n-i}\right\|+\left\|u_{N_{m}}-S_{N_{m}+i}^{*} u_{N_{m}}\right\| .
\end{aligned}
$$

Then, since $N_{m} \rightarrow \infty$ as $n \rightarrow \infty$, it follows from (52) and (54) that (55) holds obviously. Similarly, we have, for each $i \geq$ 1 ,

$$
\lim _{n \rightarrow \infty}\left\|A x_{n}-T_{i} A x_{n}\right\|=0 .
$$

Next, since $\left\{u_{n}\right\}$ is bounded, there exists a subsequence $\left\{u_{n_{i}}\right\} \subset$ $\left\{u_{n}\right\}$ such that $u_{n_{i}} \rightarrow x^{*}$ (some point in $H_{1}$ ). From (55) we have $\lim _{i \rightarrow \infty}\left\|u_{n_{i}}-S_{j} u_{n_{i}}\right\|=0$ for each $j \geq 1$. By Lemma 5 , each $S_{j}$ is demiclosed at zero, so we know that $x^{*} \in \cap_{j=1}^{\infty} F\left(S_{j}\right)$. Moreover, it follows from (48) and (50) that

$$
x_{n_{i}}=u_{n_{i}}-\gamma A^{*}\left(T_{n_{i}}^{*}-I\right) A x_{n_{i}} \rightarrow x^{*} \quad(i \longrightarrow \infty) .
$$

Since $A$ is a linear bounded operator, it yields that $A x_{n_{i}} \rightarrow$ $A x^{*}$. In view of (58) we have

$$
\lim _{i \rightarrow \infty}\left\|A x_{n_{i}}-T_{j} A x_{n_{i}}\right\|=0, \quad \forall j \geq 1 .
$$

Again since each $T_{j}$ is demiclosed at zero, we know that $A x^{*} \epsilon$ $\cap_{j=1}^{\infty} F\left(T_{j}\right)$. This implies that $x^{*} \in \Gamma$.

Note that each Hilbert space possesses Opial property, which guarantees that the weakly subsequential limit of $\left\{u_{n}\right\}$ is unique. Consequently, $\left\{u_{n}\right\}$ converges weakly to the point $x^{*} \in \Gamma$. Since $x_{n}=u_{n}-\gamma A^{*}\left(T_{n}^{*}-I\right) A x_{n}$, we know that $\left\{x_{n}\right\}$ converges weakly to $x^{*} \in \Gamma$. The proof is completed.

Remark 11. Note that, from Remark 2(3), the class of $\left(\gamma,\left\{k_{n}\right\}\right)$-asymptotically strict pseudocontractions is demiclosed at zero. Then, together with nonexpansiveness replaced by Lipschitz continuity, the two sequences of nonexpansive mappings $\left\{S_{i}\right\}$ and $\left\{T_{i}\right\}$ in Theorem 10 can be extended to $\left(\gamma,\left\{k_{n}\right\}\right)$-asymptotically strict pseudocontractions as in Lemma 7. 


\section{Conflict of Interests}

The author declares that there is no conflict of interests regarding the publication of this paper.

\section{Acknowledgments}

The author is very grateful to the referees for their useful suggestions, by which the contents of this paper has been improved. This work is supported by the General Project of Scientific Research Foundation of Yunnan University of Finance and Economics (YC2013A02).

\section{References}

[1] Y. Censor and T. Elfving, "A multiprojection algorithm using Bregman projections in a product space," Numerical Algorithms, vol. 8, no. 2-4, pp. 221-239, 1994.

[2] C. Byrne, "Iterative oblique projection onto convex sets and the split feasibility problem," Inverse Problems, vol. 18, no. 2, pp. 441453, 2002.

[3] Y. Censor, T. Bortfeld, B. Martin, and A. Trofimov, "A unified approach for inversion problems in intensity-modulated radiation therapy," Physics in Medicine and Biology, vol. 51, no. 10, pp. 2353-2365, 2006.

[4] Y. Censor, T. Elfving, N. Kopf, and T. Bortfeld, “The multiplesets split feasibility problem and its applications for inverse problems," Inverse Problems, vol. 21, no. 6, pp. 2071-2084, 2005.

[5] Y. Censor, A. Motova, and A. Segal, "Perturbed projections and subgradient projections for the multiple-sets split feasibility problem," Journal of Mathematical Analysis and Applications, vol. 327, no. 2, pp. 1244-1256, 2007.

[6] H. K. Xu, "A variable Krasnosel'skii-Mann algorithm and the multiple-set split feasibility problem," Inverse Problems, vol. 22, no. 6, pp. 2021-2034, 2006.

[7] Q. Yang, "The relaxed CQ algorithm solving the split feasibility problem," Inverse Problems, vol. 20, no. 4, pp. 1261-1266, 2004.

[8] J. Zhao and Q. Yang, "Several solution methods for the split feasibility problem," Inverse Problems, vol. 21, no. 5, pp. 17911799, 2005.

[9] T. H. Kim and H. K. Xu, "Convergence of the modified Mann's iteration method for asymptotically strict pseudo-contractions," Nonlinear Analysis: Theory, Methods \& Applications, vol. 68, no. 9, pp. 2828-2836, 2008.

[10] A. Moudafi, "A note on the split common fixed-point problem for quasi-nonexpansive operators," Nonlinear Analysis: Theory, Methods \& Applications, vol. 74, no. 12, pp. 4083-4087, 2011.

[11] A. Moudafi, "The split common fixed-point problem for demicontractive mappings," Inverse Problems, vol. 26, no. 5, Article ID 055007, 6 pages, 2010.

[12] S. S. Chang, Y. J. Cho, J. K. Kim, W. B. Zhang, and L. Yang, "Multiple-set split feasibility problems for asymptotically strict pseudocontractions," Abstract and Applied Analysis, vol. 2012, Article ID 491760, 12 pages, 2012.

[13] K. Aoyama, Y. Kimura, W. Takahashi, and M. Toyoda, "Approximation of common fixed points of a countable family of nonexpansive mappings in a Banach space," Nonlinear Analysis: Theory, Methods \& Applications, vol. 67, no. 8, pp. 2350-2360, 2007.
[14] K. Goebel and W. A. Kirk, Topics in Metric Fixed Point Theory, vol. 28 of Cambridge Studies in Advanced Mathematics, Cambridge University Press, Cambridge, UK, 1990.

[15] W. Q. Deng and P. Bai, "An implicit iteration process for common fixed points of two infinite families of asymptotically nonexpansive mappings in Banach spaces," Journal of Applied Mathematics, vol. 2013, Article ID 602582, 6 pages, 2013. 


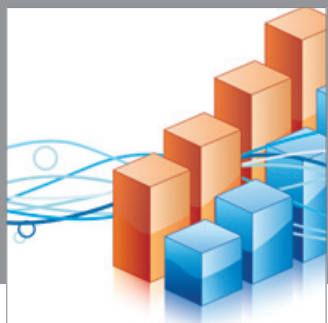

Advances in

Operations Research

mansans

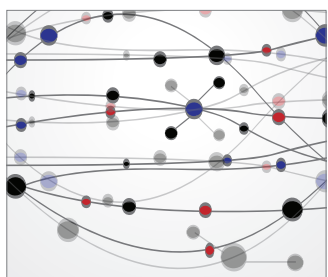

The Scientific World Journal
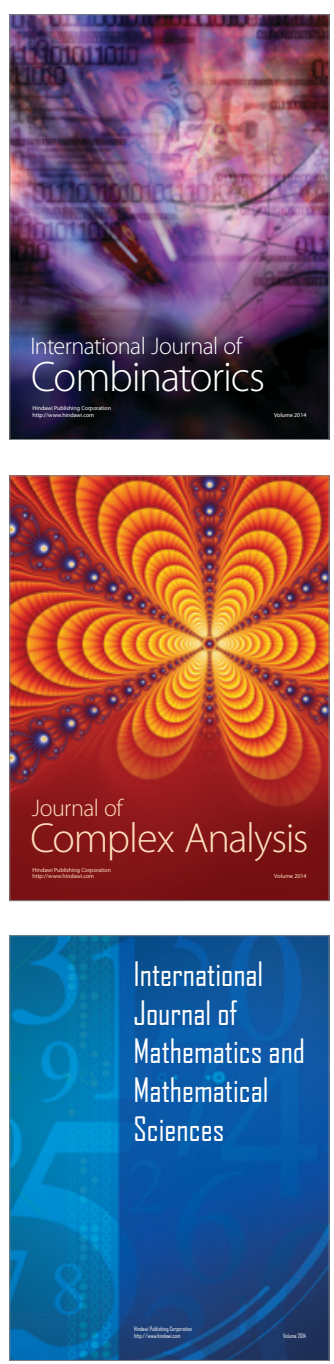
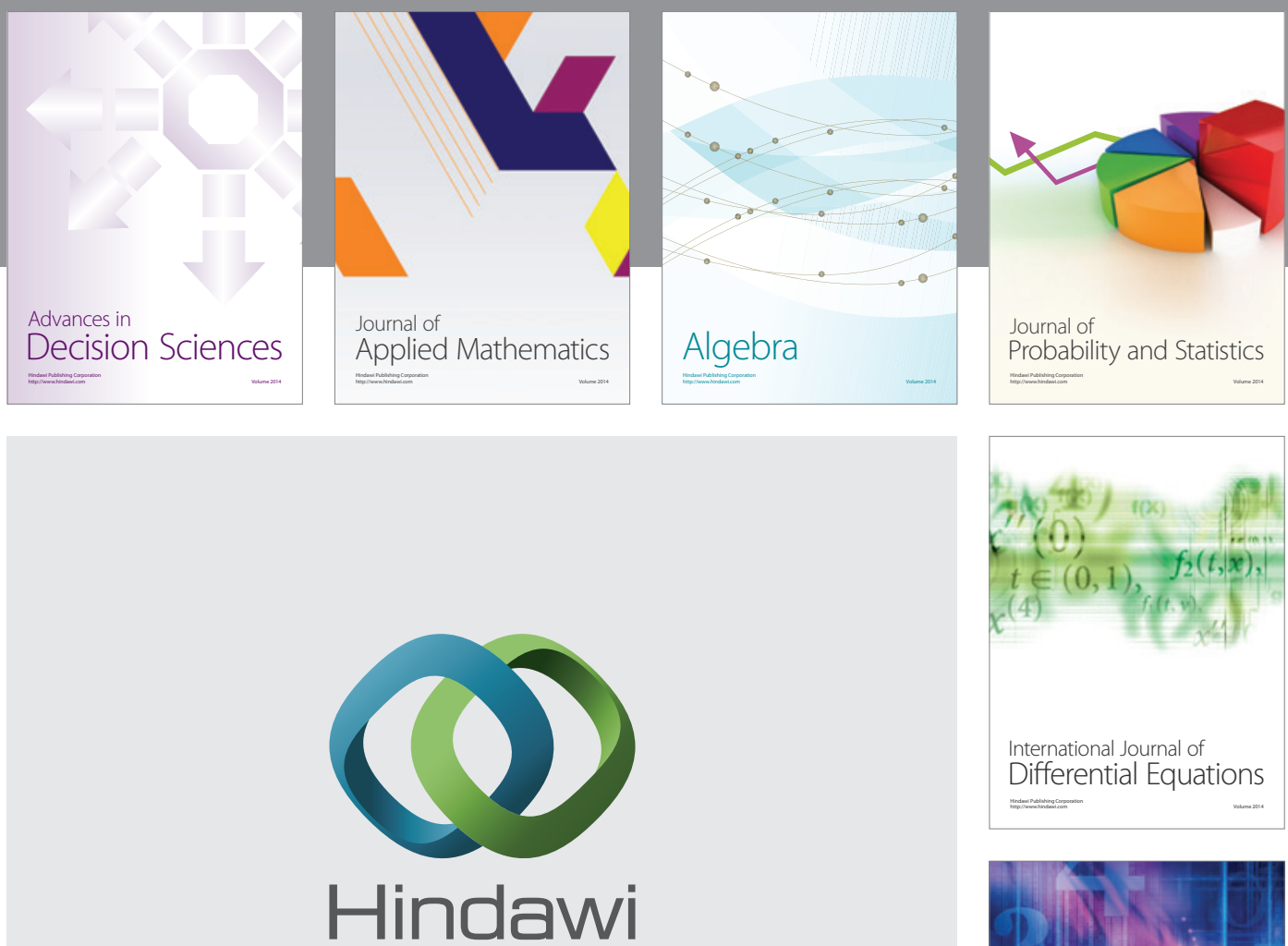

Submit your manuscripts at http://www.hindawi.com
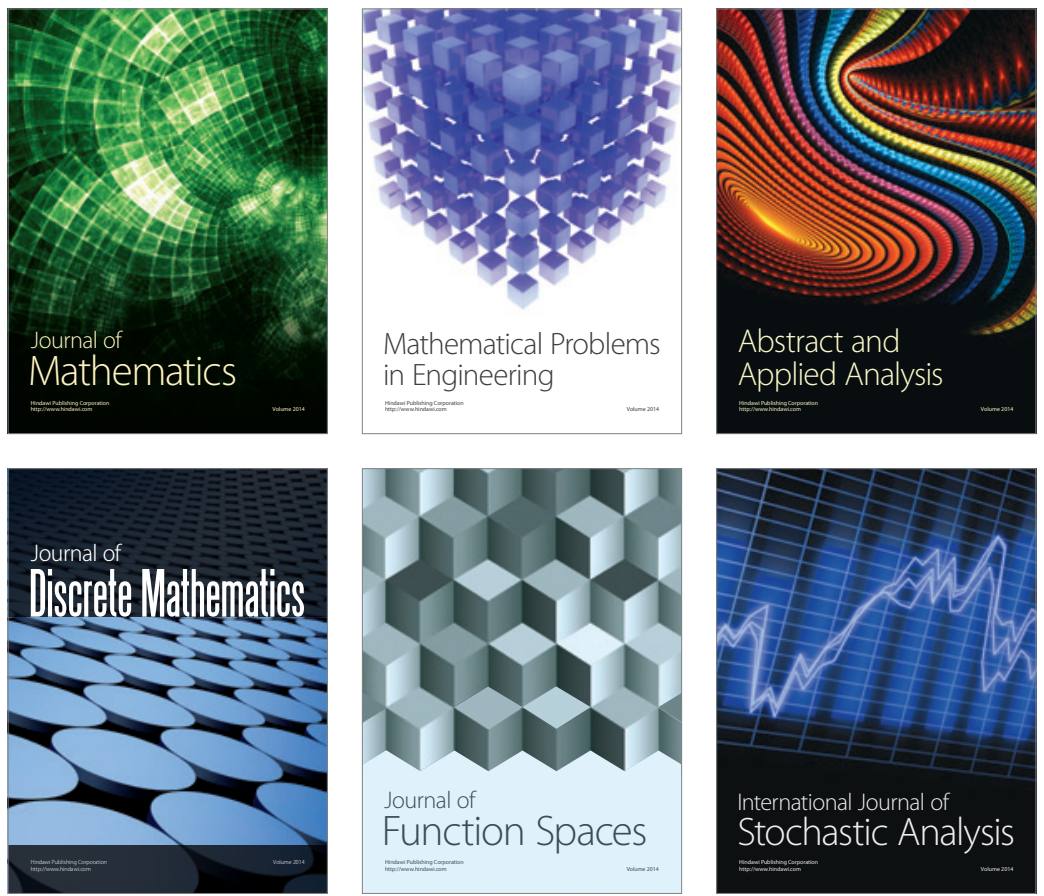

Journal of

Function Spaces

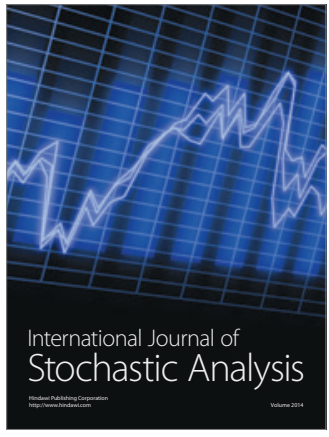

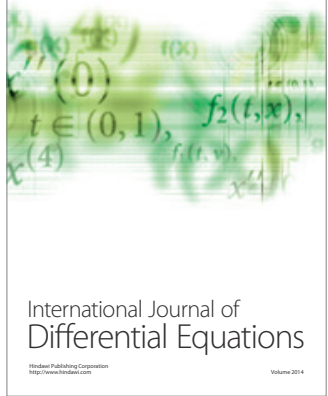
